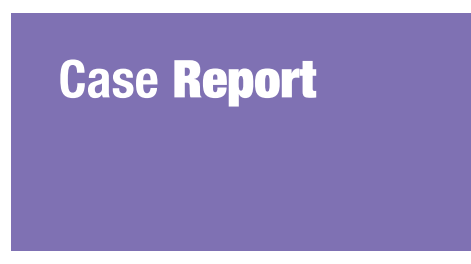

\title{
Superior Mesenteric Artery Syndrome in association with Abdominal Tuberculosis: An Eye Opener
}

Submitted: 24 Jun 2015 Accepted: 28 Aug 2016 Online: 30 Jun 2017

\author{
Shuchi Bhatt ${ }^{1}$, Biswajit Mishra ${ }^{2}$, Anupama TAndon ${ }^{1}$, Smita \\ Manchanda ${ }^{2}$, G Parthsarathy ${ }^{2}$
}

Department of Radiodiagnosis, University College of Medical Sciences and GTB Hospital, Dilshad Garden, New Delhi, India

2 Department of Radiodiagnosis, All India Institute of Medical Sciences,

New Delhi, India

To cite this article: Bhatt S, Mishra B, Tandon A, Manchanda S, Parthsarathy G. Superior mesenteric artery syndrome in association with abdominal tuberculosis: an eye opener. Malays J Med Sci. 2017;24(3):96-100. https://doi. org/10.21315/mjms2017.24.3.12

To link to this article: https://doi.org/10.21315/ mjms2017.24.3.12

\begin{abstract}
Superior Mesenteric Artery Syndrome (SMAS) is a rare clinical entity presenting as acute or chronic upper gastrointestinal obstruction. It occurs due to compression of third part of duodenum between abdominal aorta and overlying superior mesenteric artery caused by a decrease in angle between the two vessels. Rapid loss of retroperitoneal fat, in conditions leading to severe weight loss is the main factor responsible for this disorder. Superior mesenteric artery syndrome in association with abdominal tuberculosis has not been reported earlier to the best of our knowledge. Therefore, an unknown cause (SMAS) of upper gastrointestinal obstruction in a patient of abdominal tuberculosis is being presented for the first time through this case report. An imaging diagnosis of SMAS was made on contrast enhanced CT abdomen which also confirmed the clinical suspicion of abdominal tuberculosis in the patient. The patient was managed conservatively and recovered without requiring any surgical intervention for the obstructive symptoms.
\end{abstract}

Keywords: superior mesenteric artery syndrome, Wilkie's syndrome, mesenteric root syndrome, intermittent aorto-mesenteric occlusion, duodenal obstruction

\section{Introduction}

Superior Mesentric Artery Syndrome (SMAS) was first described by Rokitansky. It has a reported incidence of $0.012 \%-0.3 \%$ in one of the series of upper gastrointestinal (GI) barium studies thus, representing the rarest gastrointestinal disorder (1). It is also known as Wilkie's syndrome (2), cast syndrome, mesenteric root syndrome or intermittent aorto-mesenteric occlusion (2). A decrease in the angle and distance between the abdominal aorta and the superior mesenteric artery causes compression of the duodenum as it traverses across the spine between the two vessels. This leads to upper GI obstruction and imaging is required for making a definitive diagnosis.

\section{Case report}

A 22 year female presented with vague abdominal pain, anorexia, mild intermittent fever and weight loss for four months. She complained of recent development of postprandial heaviness, central upper abdominal pain and vomiting for past 20 days. There was no significant past medical illness reported by the patient.

On general physical examination, the patient was thin built, weighed $43 \mathrm{~kg}$ and had pallor. Local abdominal examination was unremarkable except for mild tenderness in the right iliac fossa. The routine blood examination revealed anemia (Hb-9.7 mg/dl), a normal total leucocyte count $(8400 / \mathrm{cc})$ and raised ESR 
(53 $\mathrm{mm}$ in 1 hour). Routine urine examination, liver function tests, and renal function tests were within normal limits. A clinical diagnosis of abdominal tuberculosis was suspected and patient referred for a radiological work up.

The chest X-ray was normal. Abdominal ultrasound (USG) revealed a dilated stomach and duodenum with multiple, rounded, hypoechoic, necrotic lymph nodal mass in the peri pancreatic region. Significant lymphadenopathy was also present in the mesenteric and para-aortic regions. The upper GI barium study revealed a dilated duodenum with an abrupt sharp cut off of barium column in the third part of the duodenum suggestive of SMAS (Figure 1A). Thereafter, the patient underwent an abdominal contrast enhanced computed tomography (CECT) examination to confirm the duodenum obstruction, detect its cause and for evaluating the small bowel distal to the obstruction which was not possible on barium meal follow through examination. CECT revealed a decreased aortomesenteric angle of $17.5^{\circ}$ also demonstrated in the Doppler examination (Figure 1B) in the reconstructed sagittal image (Figure 2C) and reduced distance of $5.7 \mathrm{~mm}$ between the two vessels (Figure 2A) in transverse image with resultant duodenum compression. Multiple lymph nodal groups were enlarged in the peri pancreatic, para-aortic and mesenteric regions. Lymph nodes showed central hypodensity with peripheral enhancement suggestive of necrosis (Figure 2B). Concommitent GI involvement was seen as thickening and enhancement of terminal ilium, the ileo-caecal junction along with a contracted caecum (Figure 2D).

CECT suggested a diagnosis of abdominal tuberculosis (TB) with involvement of ileo-caecal junction, necrotic abdominal lymphadenopathy and an associated SMAS.

Corroborative evidence of TB was provided by a positive mantoux test and positive IgM serology. The final diagnosis was made by USG guided fine needle aspiration cytology of the lymph nodes which revealed caseating granulomas.

The patient was started on anti-tubercular treatment (isoniazid, rifampicin, pyrazinamide) and advised a high caloric and protein diet and small frequent meals. She was advised to assume a left lateral decubitus posture after meals. A good clinical response to conservative treatment was seen as weight gain and relief of the obstructive upper GI symptoms. The follow up ultrasound and Doppler study demonstrated an increase in the aorto-mesenteric angle to $34^{\circ}$.

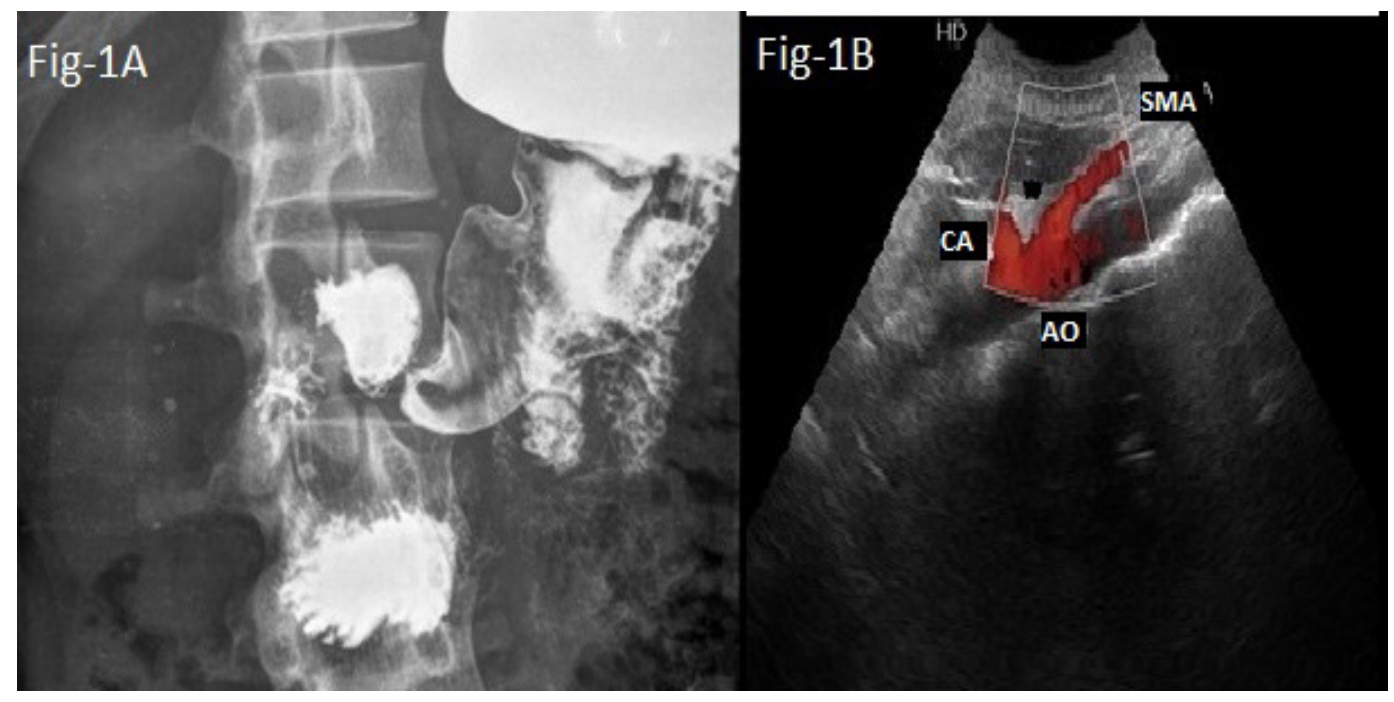

Figure 1A. Barium meal follow through showing a dilated stomach and duodenum with abrupt vertical cut off of barium column in the third part of duodenum

Figure 1B. Colour Doppler showing narrowing of aorto-mesenteric angle 


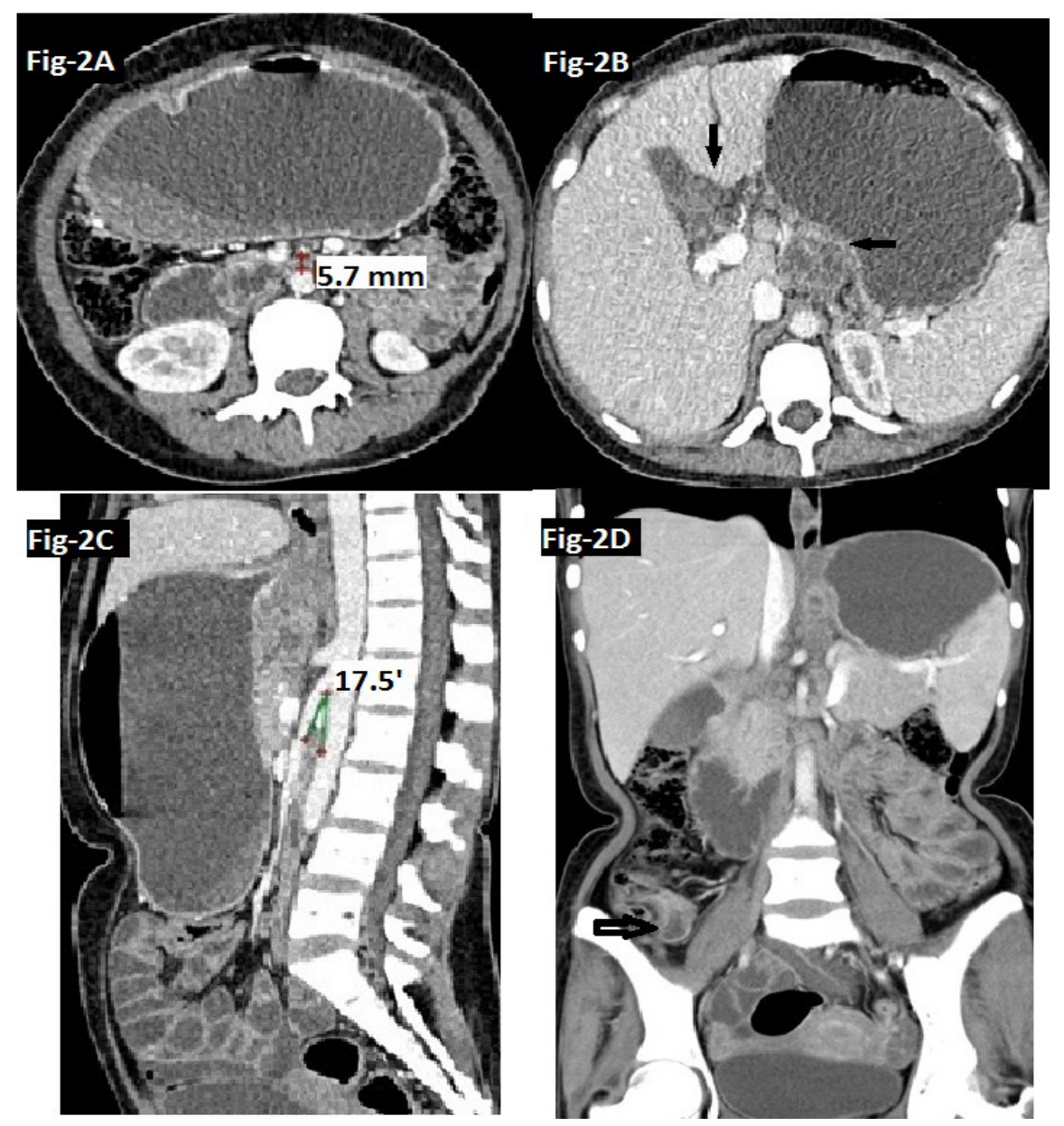

Figure 2A. CECT abdomen axial view reveals a decreased aorto-mesenteric distance of $5.7 \mathrm{~mm}$, which normally measures $10 \mathrm{~mm}-20 \mathrm{~mm}$. The third part of the duodenum is compressed between the two vessels resulting in duodenal obstruction

Figure 2B. CECT abdomen showing multiple enlarged, matted and necrotic lymph nodes in caeliac and periportal regions

Figure 2C. Sagittal reconstructed view of CECT abdomen showing aorto-mesenteric angle of $17.5^{\circ}$. Normal angle measures $38^{\circ}-56^{\circ}$. The distented stomach is also visualised

Figure 2D. CECT abdomen coronal view demonstrates thickening and enhancement of terminal ilium with a contracted caecum a finding suggestive of abdominal tuberculosis

\section{Discussion}

SMA syndrome is characterised by compression of the transverse part of the duodenum as it traverses the space between the SMA and abdominal aorta due to reduced aorta-mesenteric angle from $38^{\circ}-56^{\circ}$ to $6^{\circ}-25^{\circ}$ (3). Various disorders leading to severe weight loss like anorexia nervosa, mal-absorption, congestive heart failure, diabetes mellitus and catabolic states like burn and malignancy are the known causes.

This is the first case of abdominal tuberculosis leading to SMA syndrome.
Inflammatory stricture commonly of ileum and ileocaecal junction is the cause of subacute intestinal obstruction in abdominal TB. Duodenum obstruction due to extrinsic compression by adjacent lymph node mass or development of a duodenal stricture can occur and present as upper GI obstruction. A case of duodenum obstruction due to duodenum tuberculosis mimicking SMA syndrome has been reported in literature (4), however finding of SMA syndrome were absent in this case. A case of pulmonary TB induced cachexia causing SMA syndrome exists and reports the same etiology as 
in our case where severe weight loss was present (5).

Usually SMA syndrome presents with early satiety, post-prandial epigastric fullness, nausea and vomiting of partially digested food and malnutrition due to persistent vomiting thus leading to a vicious cycle.

The diagnosis of SMA syndrome rests on clinical suspicion and is diagnosed by demonstration of obstruction at the third (transverse) part of the duodenum due to compression by the superior mesenteric artery, on imaging. Barium studies are done to demonstrate the level and cause of obstruction. The finding of abrupt sharp cut off of the barium column in the dilated third part of duodenum is a characteristic finding. Fluoroscopy demonstrates to and fro peristalsis proximal to obstruction with slow, delayed passage of barium into the collapsed distal loops which improves in the left lateral decubitus position. Few investigators have suggested the use of hypotonic duodenography for demonstration of SMA syndrome when conventional barium studies fail to show the pathology (6).

CECT abdomen can directly demonstrate the reduced distance and angle between the SMA and abdominal aorta and the resultant duodenum compression. The normal aortomesenteric angle of $38^{\circ}-56^{\circ}$ which when reduced to $6^{\circ}-25^{\circ}$ causes duodenal compression. The normal aorta-mesenteric distance is $10 \mathrm{~mm}$ $20 \mathrm{~mm}$ which is reduced to $2 \mathrm{~mm}-8 \mathrm{~mm}$ in SMA syndrome. There is a strong correlation between clinical diagnosis of SMA syndrome and the CT/ Doppler measurements (angle and distance) between the aorta and superior mesenteric artery. A distance of $8 \mathrm{~mm}$ is $100 \%$ sensitive and specific while an aorto-mesenteric angle of $22^{\circ}$ is $42.8 \%$ sensitive and $100 \%$ specific for the diagnosis of SMA syndrome (7). CT angiography, magnetic resonance imaging and color Doppler techniques (Figure 1B) can be used to measure the aorto-mesenteric angle at the point of origin of the SMA from the aorta. Ultrasonography and CT can also be used to rule out other causes of duodenal compression like enlarged lymphnodes or tumor.

Treatment is usually conservative aiming at treating the cause, decompression of bowel through naso-gastric tube drainage, hyper alimentation and advising small frequent feeds. Prokinetic drugs and decubitus posture to facilitate duodenum emptying can be tried. Surgery is undertaken in long standing cases not responding to conservative management (5).
Open or laparoscopic duodeno-jejunostomy is the procedure of choice and is effective in $90 \%$ of cases. Cleavage of ligament of treitz is another option to relieve duodenum obstruction.

Severe weight loss associated with abdominal tuberculosis can cause SMA syndrome as in our patient. Development of upper GI obstructive symptoms should alarm the clinician of this entity. Such patients should be investigated with a contrast CT examination of the abdomen for demonstration of the level and cause of obstruction. The orientation of the SMA with respect to the aorta can be easily judged on the reconstructed images for diagnosis of SMA syndrome. Accurate timely diagnosis and appropriate treatment increases the chances of success of conservative treatment. Non response to medical management is an indication for surgery.

\section{Authors' Contributions}

Conception and design: SB

Analysis and interpretation of the data: SB, BM

Drafting of the article: BM, SM, GP

Critical revision of the article for important intellectual content: SB

Final approval of the article: SB, AT, SM

Collection and assembly of data: GP

\section{Correspondence}

Dr Biswajit Mishra

MD (Aryabhatt Knowledge University)

Senior resident

Department of Radiodiagnosis

All India Institute of Medical Sciences

Ansari Nagar,

New Delhi 110029, India.

Tel: +918527160797

Fax: +91-11-22590495

E-mail: biswajitmishramkcg@gmail.com

\section{References}

1. Bedoya R, Lagman SM, Pennington GP, Kirdnual A. Clinical and radiological aspects of superior mesenteric artery syndrome. J Fla Med Assoc. 1986;73:686-689.

2. Ansari WA, Shaikh AS, Jamadar NM. Superior mesenteric artery (Wilkie's) syndrome. Bombay Hospital Journal. 2009;51:503-507. 
3. Welsch T, Buchler MW, Kienle P. Recalling superior mesenteric artery syndrome. Digest Surg. 2007;24:149-56.

4. Bakshi GD, Ansari WA, Chavan PR, Sarangi S. Duodenal tuberculosis mimicking superior mesenteric artery syndrome. Bombay Hospital Journal. 2005;47:286-288.

5. Limaye CS, Karande SP, Aher SP, Patel KA. Superior mesenteric artery syndrome secondary totuberculosis induced cachexia. $J$ of Assoc Physicians. 2011;58:668-669.
6. Lukes PJ, Rolny PJL, Nilson AE. Diagnostic value of hypotonic duodenography in superior mesenteric artery syndrome. Acta Chir Scand. 1978;144:39-43.

7. Unal B, Aktas A, Kemal G, Bilgili Y, Guliter S, Daphan C, Aydinuraz K. Superior mesenteric artery syndrome-CT and ultrasonography finding. Diag Interv Radiol. 2005;11(2):90-95. 